\title{
Empirical Studies on Topographical Influences on Crbc/Akbc Terrestrial Television Stations' Signals in Akpabuyo Local Council Area, Nigeria
}

\author{
Donatus Enang Bassey ${ }^{*}{ }^{\circledR}$, Godwin 0. Effiong1, Ofem U. Omini², Effiong 0. Obisung ${ }^{3}$ \\ ${ }^{1}$ Electronics/Computer Technology Unit, Faculty of Engineering, University of Calabar, Calabar, Nigeria \\ ${ }^{2}$ Electrical Engineering Department, Cross River University of Technology, Calabar, Nigeria \\ ${ }^{3}$ Physics Department, Faculty of Physical Sciences, University of Calabar, Calabar, Nigeria \\ Email: ^basseyde1@yahoo.com
}

How to cite this paper: Bassey, D.E., Effiong, G.O., Omini, O.U. and Obisung, E.O. (2019) Empirical Studies on Topographical Influences on Crbc/Akbc Terrestrial Television Stations' Signals in Akpabuyo Local Council Area, Nigeria. Journal of Computer and Communications, 7, 50-71.

https://doi.org/10.4236/jcc.2019.79005

Received: August 16, 2019

Accepted: September 17, 2019

Published: September 20, 2019

Copyright (๑) 2019 by author(s) and Scientific Research Publishing Inc. This work is licensed under the Creative Commons Attribution International License (CC BY 4.0).

http://creativecommons.org/licenses/by/4.0/

\begin{abstract}
Dissemination of information to citizens is a relevant component of governance. Expectedly, viewers tuned to broadcast stations within and outside their localities, expecting their receivers to faithfully reproduce the exact features of the transmitted signal. Akpabuyo is a dense forest zone near the creeks leading to the Atlantic ocean in Cross River State, Nigeria. The location has distinct environmental characteristics that made Akpabuyo Area Council, a challenging location to propagate electromagnetic waves; and therefore recipe for further investigation. Radio frequency analyzer, with 24 channels spectrum, ranging between $46-870 \mathrm{MHz}$ (model: RO.VE.R.-“DLM3-T") was deployed to capture signals from terrestrial television stations (TV). CATV measured signal of $\mathrm{TV}$ stations in $\mathrm{dB}, \mathrm{dB} \mu V$ and $\mathrm{dBmV}$. Its frequency ranged from $40-860 \mathrm{MHz}$; while varying from channel 1 to channel 69. Measurements taken from Akpabuyo L.G.A. showed the following results: the signal strength received from VHF Channel 11 ranged from $20 \mathrm{~dB} \mu V$ to $49 \mathrm{~dB} \mu V$. From recorded empirical statistics from the study, $50 \%$ of the area received signal from this station above $30 \mathrm{~dB} \mu V$, while other regions had signals below this value; representing the fringe zone of the frequency. The results obtained from the study relatively showed acceptance with Egli's model. The study recorded a steady fluctuation between $17 \mathrm{~dB} \mu V$ and $19 \mathrm{~dB} \mu V$ from both propagating stations. However, Channel 27 signal at $519 \mathrm{MHz}$, had very weak signal coverage in Akpabuyo Local Government Area; with signal strength dropped to as low as $13 \mathrm{~dB}$ in many parts of the rural area. The study discovered that the state's broadcasting stations, both at UHF and VHF channels did not transmit successfully across this densely forest (rural) location. Remedial measures such as installing Repeater stations at different locations as signal booster were recommended.
\end{abstract}




\section{Keywords}

Akamkpa, Biase, Broadcast Station, Channels, Propagation Models, Signal, Television Stations, UHF/VHF Signals

\section{Introduction}

The broadcast industry plays a major role in information dissemination to citizens. It is one major out-fit where every citizen can be informed of major events worldwide; even when they cannot afford to own a receiver-station. Thus, the availability of television in almost every home cannot be questioned. According to records, about 1.4 billion of the world's population own television-set; indicating that one out of every five people owns a television. Analysis from research studies shows that China tops the chart with $28.2 \%$, followed by US with $15.5 \%$ [1].

One of the transmission media of information technology is done through VHF (Very High Frequency) and UHF (Ultra High Frequency) bands. These frequency bands are assigned to stations for terrestrial broadcasting. As viewers tuned to broadcast stations within and outside their localities, they expect the television to faithfully reproduce the exact audio and video features transmitted by the broadcasting station's transmitter: the structural content or shape of object, the tonal content or relative brightness, the motion of the object or kinematic content, sound, color or chromatic content and lastly the stereoscopic content also known as perspective [2]. These contents are direct function of how good the signal is at the point of reception. In other word, they reflect the signal strength at such point. The signals are electromagnetic in nature and as such are subject to natural or man-made phenomena. Factors like the presence of hills, buildings, vegetation and the atmosphere have been found to have a great influence on the propagation of this signal in different regions. The areas of reception have been classified into primary, secondary and fringe service area [3] [4]. Based on environmental influences, the signal may decreases from primary to fringe zone.

Wave could be seen as an oscillation or vibration or disturbance that is characterized by energy transfer that moves through space or object [5].

\section{Statement of the Problem}

Propagation loss has been established to be inevitable as the distance between the transmitting antenna and the receiving antenna increases. As viewers tuned to broadcast stations within and outside their localities, they expect the television to faithfully reproduce the exact audio and video features transmitted by the broadcasting station's transmitter (Kennedy and Davies, 1993) [3]. Audio signal quality can deteriorate without much impact on the received audio signal; as well as message. However, visual signals are highly sensitive to fluctuations, and 
therefore, easily noticed to the discomfort of the viewer. This research study is therefore focused on the UHF/VHF visual signal distortions. It carried out measurements and analyses of signal strength levels from two propagating television stations (UHF and VHF): Akwa Ibom Broadcasting Corporation (AKBC) and Cross River Broadcasting Corporation (CRBC). This is with a view to establishing the primary, secondary and fringe service areas of these signals at different strategic locations, and topographical influences on the delivered signal strength levels in the Akpabuyo Local Government Area, Nigeria. Results from the study shall be of immense benefit to both the Broadcasting managers and residents within the coverage area.

\section{Theoretical Frame Work}

Waves have two main types and they are mechanical and electromagnetic waves. Whereas mechanical waves require material medium for its propagation, electromagnetic waves requires no material medium for its propagation. When an electric current flows through a conductor, it produces a time-varying electric field which in turn acts as a source of magnetic field. These two fields created can sustain and interact with each other giving rise a special form of energy known as electromagnetic wave [6]. According to Maxwell's equations, both electric and magnetic fields which form the electromagnetic disturbance are sinusoidal functions of time and position, and characterized by frequency and wavelength. These equations and direction of propagation define the basic principle undergirding the operation of electromagnetic wave as a combination of Gauss's law of electric fields, Gauss's law of magnetic fields, Ampere's law and Faraday's law [6]. The equations are as follows:

$$
\oint E \cdot \mathrm{d} A=Q_{\text {encl }} / \epsilon_{\circ}
$$

Equation (1) implies that the surface integral of $E$ over any closed surface equals $1 / \epsilon$ multiplied by the total charge $Q_{\text {enc }}$ where $E$ is the electric field and $A$ represents the area.

$$
\oint B \cdot \mathrm{d} A=0 .
$$

Equation (2) states that the surface integral of $B$ over any closed surface is zero, where $B$ is the magnetic field.

$$
\oint B \cdot \mathrm{d} l=\mu\left(i_{\mathrm{c}}+\varepsilon_{\mathrm{o}} \frac{\mathrm{d} \Phi}{\mathrm{d} t} E\right)
$$

This is also known as Ampere's law. It implies that both conduction and displacement current act as sources of magnetic field; where $\varepsilon_{\circ}$ is the permittivity of free space, $\mu_{\circ}$ the permeability of free space, and $\Phi E$ the electric flux.

$$
\oint E \cdot \mathrm{d} l=-\frac{\mathrm{d} \Phi}{\mathrm{d} t} E
$$

Equation (4) is Faraday's law and implies that a changing magnetic flux induces an electric field. The Heaviside version of this equation using Laplace 
transform is shown in Equations (5) to (8) below:

$$
\begin{gathered}
\nabla \cdot E=0 \\
\nabla \times E=-\frac{\partial B}{\partial t} \\
\nabla \cdot B=0 \\
\nabla \times E=\frac{\mu_{\mathrm{o}} \epsilon_{\mathrm{o}} \partial E}{\partial t}
\end{gathered}
$$

Deploying the curl of curl to these equations, we obtain Equations (9) and (10) as presented below:

$$
\begin{aligned}
& \nabla \times(\nabla \times E)=-\frac{\partial}{\partial t} \nabla \times B=-\frac{\mu_{\circ} \epsilon_{\mathrm{o}} \partial^{2} E}{\partial t^{2}} \\
& \nabla \times(\nabla \times B)=-\frac{\partial}{\partial t} \nabla \times E=-\frac{\mu_{\mathrm{o}} \epsilon_{\mathrm{o}} \partial^{2} E}{\partial t^{2}}
\end{aligned}
$$

Further to the above, applying the vector identity, Equation (11) is derived as:

$$
\nabla \times(\nabla \times V)=\nabla \cdot(\nabla \cdot V)-\nabla^{2} V
$$

where $V$ is any vector function in space. And Equation (12),

$$
\nabla^{2} V=\nabla \cdot(\nabla \cdot V)
$$

where $\nabla V$ is a dyadic which when operated on by the divergence operator $\nabla$. yields a vector. Since

$$
\begin{aligned}
& \nabla \cdot E=0 \\
& \nabla \cdot B=0
\end{aligned}
$$

Then, the first term on the right in the identity vanishes and the wave equations are obtained as presented below:

$$
\begin{aligned}
& \frac{\partial^{2} E}{\partial t^{2}}-c_{\circ}^{2} \cdot \nabla^{2} E=0 \\
& \frac{\partial^{2} B}{\partial t^{2}}-c_{\circ}^{2} \cdot \nabla^{2} B=0
\end{aligned}
$$

where $C$ in Equation (15):

$$
c_{\circ}=\frac{1}{\sqrt{\mu_{\mathrm{o}} \epsilon_{\mathrm{o}}}}=2.99792458 \times 10^{8} \mathrm{~m} / \mathrm{s}
$$

is the speed of light in free space.

Each of these radiations has both frequency and wavelength range called bands, and are expressed below [7]: Radio Waves: $10 \mathrm{~cm}$ to $10 \mathrm{~km}$ wavelength; Microwaves: $1 \mathrm{~mm}$ to $1 \mathrm{~m}$ wavelength. $\left(1 \mathrm{GHz}=10^{9} \mathrm{~Hz}\right)$; P band: $0.3-1 \mathrm{GHz}$ $(30-100 \mathrm{~cm})$; L band: $1-2 \mathrm{GHz}(15-30 \mathrm{~cm})$; S band: $2-4 \mathrm{GHz}(7.5-15 \mathrm{~cm})$; C band: 4 - $8 \mathrm{GHz}(3.8$ - $7.5 \mathrm{~cm})$; $\mathrm{X}$ band: 8 - $12.5 \mathrm{GHz}(2.4-3.8 \mathrm{~cm})$; $\mathrm{Ku}$ band: 12.5 - $18 \mathrm{GHz}(1.7$ - $2.4 \mathrm{~cm})$; K band: 18 - $26.5 \mathrm{GHz}(1.1-1.7 \mathrm{~cm})$; Ka band: 26.5 - $40 \mathrm{GHz}(0.75-1.1 \mathrm{~cm})$; Infrared: 0.7 to $300 \mu \mathrm{m}$ wavelength; Near Infrared (NIR): 0.7 to $1.5 \mu \mathrm{m}$; Short Wavelength Infrared (SWIR): 1.5 to $3 \mu \mathrm{m}$; Mid 
Wavelength Infrared (MWIR): 3 to $8 \mu \mathrm{m}$; Long Wavelength Infrared (LWIR): 8 to $15 \mu \mathrm{m}$; Far Infrared (FIR): longer than $15 \mu \mathrm{m}$; Visible Light: It ranges from about $400 \mathrm{~nm}$ (violet) to about $700 \mathrm{~nm}$ (red); Red: 610 - $700 \mathrm{~nm}$; Orange: 590 $610 \mathrm{~nm}$; Yellow: 570 - $590 \mathrm{~nm}$; Green: 500 - $570 \mathrm{~nm}$; Blue: 450 - $500 \mathrm{~nm}$; Indigo: 430 - $450 \mathrm{~nm}$; Violet: 400 - $430 \mathrm{~nm}$; Ultraviolet: 3 to $400 \mathrm{~nm}$; X-Rays and Gamma Rays; Nakanishi (2013) indicated that there are other approaches that have the ability to track the passage of electromagnetic waves [7]. Figure 1, below is a graphical presentation of the Electromagnetic spectrum.

The radial electric field surrounding such electron is given by the Equation (16) below:

$$
E r=\frac{1}{4 \pi \varepsilon_{0}} * \frac{e}{r^{2}}
$$

The condition stated by Equation (16) holds only for a stationary electron; but changes when such an electron is moving. Example: a current-carrying wire. The moving electron which produces current is also responsible for the magnetic field around it.

According to [6], any new field (electromagnetic radiation) produced by an accelerating electron depends on the acceleration of the electron and the reciprocal of the distance of the electron from the nucleus. This theory is illustrated in Equation (17) below:

$$
E_{\theta}=e * \frac{1}{4 \pi \varepsilon_{0}} * \frac{\ddot{r} \sin \theta}{c^{2} r}
$$

where $E_{\theta}$ is the pulse of electromagnetic radiation, $e$ is the electronic charge, $r$ is the acceleration, $c$ is the speed of light, $r$ the distance from the nucleus, and the angle between the changing fields. The amount of radiation leaving the system also depends of the length of the current-carrying conductor and the wavelength of the current flowing through it. Base on this, electromagnetic radiation can be described as an energy that is transmitted in form of electromagnetic wave either through space or a material medium.

\subsection{Attenuation of Electromagnetic Radiations}

This is one of the major setbacks in the propagation of electromagnetic waves when it leaves its source. Researchers have shown that electromagnetic waves get attenuated as they travel outwardly. Attenuation can be referred to as a reduction in the intensity of propagated electromagnetic waves. Attenuation of electromagnetic radiation in space obeys the inverse-square law which indicates that power density reduces fairly rapidly with distance from the source. This means that signal attenuation, is proportional to the square of the distance travelled by the wave [2]. The attenuation of field intensity is given by Equation (18):

$$
\alpha_{E}=\frac{\sqrt{\frac{30 P_{t}}{r_{1}}}}{\sqrt{\frac{30 P_{t}}{r_{2}}}}=20 \log \frac{r_{1}}{r_{2}}
$$




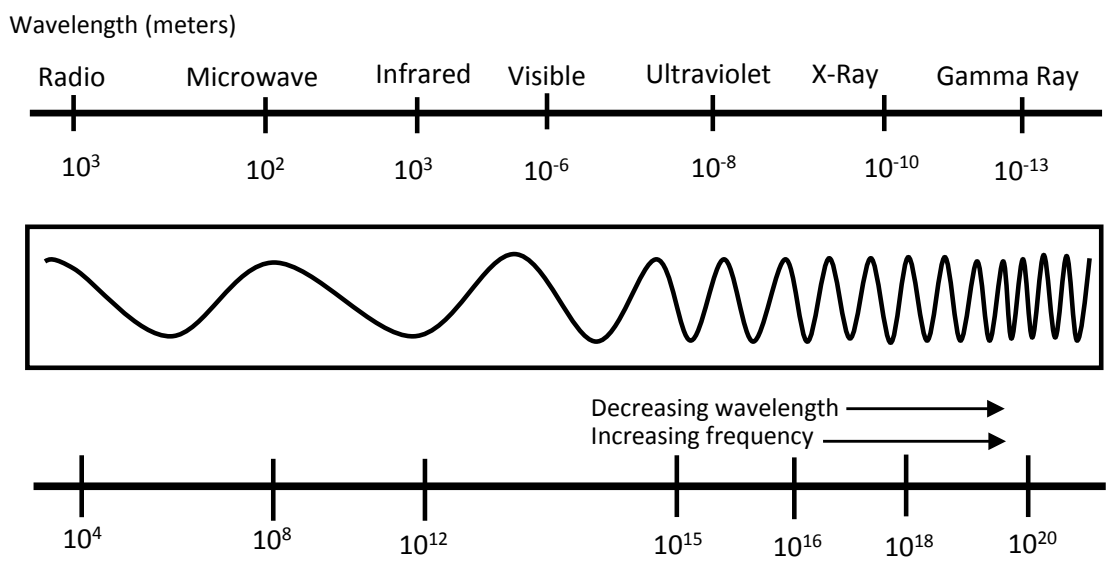

Figure 1. The electromagnetic spectrum.

where $\alpha_{E}$ is the field intensity attenuation, $P_{t}$ the transmitted power, $r_{1}$ and $r_{2}$ are distances from the source of electromagnetic waves with $r_{2}$ greater than $r_{1}$. This implies that at a distance of $2 r$ from the source of the electromagnetic waves, the field intensity drops by $6 \mathrm{~dB}$.

\subsection{Modulation Processes}

Propagating signals undergo modulation processes; such as amplitude, Frequency and phase modulation. The general form of an amplitude modulated wave is presented in Equation (19):

$$
f(t)=A \sin \left(2 \pi f_{c} t\right)+A m / 2\left[\sin 2 \pi\left(f_{c}+f_{m}\right) t+\varnothing\right]+\left[\sin 2 \pi\left(f_{c}-f_{m}\right) t-\varnothing\right]
$$

where $A$ and $f_{c}$ are the carrier amplitude and frequency, respectively. $m$ is the modulation index, $f_{m}$ and $\varnothing$ are the frequency and phase of the baseband signal respectively. From the above equation, the modulation has three components which are the carrier wave and the two sidebands with frequencies above and below the carrier frequency [6]. Frequency modulation (FM), is an alternative to $\mathrm{AM}$, in order to achieve a radio transmission with much resistant to noise. It is a form of angle modulation in which the frequency of the carrier signal is varied by the modulating the baseband signal while its amplitude and phase remain constant. The general form of an instantaneous frequency modulated wave is given by in Equation (20).

$$
f=f_{c}\left(1+k v_{m} \cos w_{m} t\right)
$$

where $f_{c}=$ carrier frequency, $k=$ proportionality constant, $v_{m} \cos =$ instantaneous modulating voltage. The modulation index of an FM signal is given in Equation (21):

$$
m=\frac{\Delta f}{f_{m}}
$$

where $\Delta f$ is the maximum deviation of the instantaneous frequency, and $f_{m}$ is the maximum frequency of the modulating signal.

Phase modulation PM is another form of angle modulation; besides FM where 
the phase of the carrier signal is varied by the modulating signal. In phase modulation, the variation in the carrier phase is proportional to the baseband signal, while its amplitude and frequency are constant during the process. The general form of a phase modulating wave is given as

$$
v=A_{c} \sin \left(w_{c} t+m_{(t)}+\varnothing_{c}\right)
$$

where $A_{c}$ and $w_{c}$ are the amplitude and angular of the carrier signal respectively, $m_{(t)}$ is the modulating signal and $\varnothing_{c}$ is the phase of the carrier signal being modulated.

[6] presented an efficient realization of a filtered multi-tone (FMT) modulation system and its orthogonal design. FMT modulation was seen as a Discrete Fourier Transform (DFT) modulated filter bank (FB). It generalized the popular orthogonal frequency division multiplexing (OFDM) scheme by deploying frequency continued sub channel pulses. They considered the design of an orthogonal FMT system and exploited the third realization which allowed simplifying the orthogonal FB design and obtained a block diagonal system matrix with independent sub blocks. [6] modified a Michelson interferometer to produce a wide-band FM signal having a center frequency of $80 \mathrm{MHz}$. According to [6], assembling many radio stations in the same vehicle or network of radios is likely to induce self-interference. They analyzed the influence of an Adjacent Channel Interference (ACI) and white Gaussian noise of FM communication, and established an ACI model of FM communication system and this enabled them to evaluate the influence of $\mathrm{ACI}$ interference which they realized by computing the distortion of the speech signal demodulated. Also in 2007, McCue examined the problem of radio-frequency interference (RFI) between radars using linear-FM pulses. According to him, in most of the scenarios he considered, the RFI remain the same as if the FM were not present while in other cases it became very easy to evaluate the peak response of an un-weighted receiver by mere calculation. $\mathrm{He}$ finally generated expressions for the RFI and showed that if one knows peak of the response, the effect of the weighting can as well be approximated by some simple expressions. [2] discovered a low profile polarized cavity-backed antennas using substrate integrated waveguide (SIW) techniques. According to the research, SIW and half-mode (HMSIW) techniques in antenna designs allows for low-profile cavity-backed structure using low-cost standard printed circuit board process. They used two single fed low-profile cavity-backed antennas and an antenna array for circular polarization to achieve their results. [6] both proposed a low cost printed dipole antenna as a perfect feed for prime focus reflectors. The dipoles were arranged such that their arms were oppositely placed in a dielectric substrate which is fed by a microstrip line. After this, they realized an impedance bandwidth of $16.5 \%$ with 2.5 dielectric constant substrate and an overall dimension of $60 \times 60 \times 1.58 \mathrm{~mm}^{3}$ at $3 \mathrm{GHz}$. The beamwidth of any antenna refers to the angle between half power point $(3 \mathrm{~dB})$ and the maximum power point in the radiation lope. He used a $7 \times 7$ rectangular ring unit metal surface and a single-feed, circular polarized rectangular slotted patch antenna to 
enhance bandwidth. They realized after simulation that while using the metal surface that the bandwidth of the circular polarized antenna increased seven times that of a normal antenna.

\subsection{Electric Field Strength}

The intensity or power of transmitted signal from a transmitting station received by an antenna at a different location is referred to as the electric field strength. Electric field strength is measured in $\mathrm{dB}$ millivolt per metre $(\mathrm{dBmV} / \mathrm{m})$ or $\mathrm{dB}$ microvolt per metre $(\mathrm{dBuV} / \mathrm{m})$. In 1998, Werner and Emders researched on an alternative means of calibration of electric field sensor which will enable a smooth computation of coupling errors which was not dealt with by previous methods of calibration like the use of closed transverse electromagnetic (TEM) wave. The new method involves the superposition of radiated and guided wave, and this presented an easy assessment of the coupling factor, a key index for computation of electric field strength, calibrations of sensors and antennas. Ajayi (2005) [3], used ground conductivity to predict the field strength for medium frequency transmitter in Ondo state, Nigeria. He measured ground conductivity using electrical resistivity method that is Wenner arrangement of electrodes and obtained the average ground conductivity for different soil types he used. The values were $3.02 \pm 0.29 \mathrm{mS} / \mathrm{m}$. He then used these values with other propagation models to establish suitable propagation curves for that radio station, thus predicting the field strength at different locations all over the geographical coverage of the transmitter.

\subsection{Pathloss and Models}

The intensity of radio wave leaving a transmitting station is found to differ in value at some locations away from the transmitter. This means that not all the intensity or signal strength that is transmitted by a station is being received at measured distances from the transmitter. Some percentages are lost in the propagation path. This drop in signal power as the radio wave propagates through space is known as path loss. A lot of factors are responsible for this phenomenon such as poor terrain and environment like the rural, sub-urban or urban areas, presence of hills, mountains, foliage etc and transmitter-antenna distance. Other factors include the height and location of antennas, diffractions and absorption of the electromagnetic waves. Path loss models on the other hand are experimented mathematical expressions used when illustrating radio wave propagation as a function of frequency, distance and some conditions. There are different models in existence for different conditions of the atmosphere, terrain, paths, obstructions, etc. The models for outdoor attenuations include:

1) Weissberger's modified exponential model. It is a radio wave propagation model for predicting path loss by foliage. It has a frequency range of $230 \mathrm{MHz}$ to $95 \mathrm{GHz}$ with foliage depth of $400 \mathrm{~m}$. It is a reviewed ITU (International Telecommunication Union) model for Exponential Decay (MED) created in 1982 [8]. 
Weissberger's model is formally expressed as:

$$
L= \begin{cases}1.33 f^{0.284} d^{0.586}, & \text { if } 14<d \leq 400 \\ 0.45 f^{0.284} d, & \text { if } 0<d \leq 14\end{cases}
$$

where $L=$ the loss due to foliage in decibel $(\mathrm{dB}) ; F=$ the transmission frequency gigahertz $(\mathrm{GHz}) ; d=$ the depth of foliage along the path in meters.

2) Early ITU model. It is also a radio propagation model for predicting loss due to foliage and was adopted in late 1986 [8]. It has no specified frequency range and no foliage depth and it is expressed as:

$$
L=0.2 f^{0.3} d^{0.6}
$$

where $f$ and $d$ are defined as above; and $L=$ path loss in $\mathrm{dB} ; F=$ transmission frequency in megahertz $(\mathrm{MHz}) ; d=$ depth of the foliage.

3) Egli model: This is a model sourced from UHF and VHF television transmissions data in several large cities and suitable for cellular communication on irregular terrain. It predicts the path loss for outdoor line-of-sight transmissions and expressed as follows [9]:

$$
P_{R 50}=0.668 G_{R} G_{m}\left[\frac{h_{B} h_{m}}{d^{2}}\right]^{2}\left[\frac{40}{f}\right]^{2} P_{T}
$$

where $P_{R 50}=50^{\text {th }}$ percentile receive power $(\mathrm{W}) ; P_{T}=$ Transmit Power $(\mathrm{W}) ; G_{B}=$ Absolute gain of the mobile station antenna; $h_{B}=$ Height of the base station antenna (m); $h_{m}=$ Height of the mobile station antenna $(\mathrm{m}) ; d=$ Distance from base station antenna $(\mathrm{m}) ; f=$ frequency of transmission $(\mathrm{MHz})$.

4) ITU terrain model: This provides a method to predict the medium path loss for a telecommunication link and its prediction is based on the height of the path blockage [9]. It is applicable on any terrain and expressed as follows:

$$
A=10-20 C_{N(37)} ; C_{N}=\frac{h}{F i} ; h=h_{L}-h_{o} ; F_{1}=17.3 \sqrt{\frac{d_{1} d_{2}}{f d}}
$$

$A=$ Additional loss (in excess of free - space loss) due to diffraction (dB); $C_{N}$ = Normalized terrain clearance; $h=$ The height difference (negative in the case that the LOS path in completely obscured) (m); $h_{L}=$ Height of the line-of-sight link (m); $h_{o}=$ Height of the obstruction (m); $F_{1}=$ Radius of the First Fresnel zone $(\mathrm{m}) ; d_{1}=$ Distance of obstruction from one terminal $(\mathrm{m}) ; d_{2}=$ Distance of obstruction from the other terminal (m); $f=$ Frequency of transmission $(\mathrm{GHz}) ; d$ $=$ Distance from transmitter to receiver $(\mathrm{km})$.

5) Young model: It is an appropriate model for predicting path loss in cellular communications in large cities with tall structures. It has a frequency range of $150 \mathrm{MHz}$ to $3700 \mathrm{MHz}$ and it is expressed as presented in Equation (27) [10]:

$$
L=G_{B} G_{m}\left(\frac{h_{B} h_{m}}{d^{2}}\right)^{2} \beta
$$

$L=$ path loss in (dB); $G_{B}=$ gain of base transmitter (dB); $G_{m}=$ gain of mobile 
transmitter $\left(\mathrm{dB} ; h_{B}=\right.$ height of base station antenna $(\mathrm{m}) ; h_{m}=$ height of mobile station antenna $(\mathrm{m}) ; d=$ line distance $(\mathrm{Km}) ; \beta=$ clutter factor.

6) Okumura model: This is used mostly in the cities where there are many urban structures, but not many tall blocking structures. It has a frequency range of $150-1920 \mathrm{MHz}$, a base station antenna height between $30 \mathrm{~m}$ and $1000 \mathrm{~m}$ and link distance between $1 \mathrm{Km}$ and $100 \mathrm{~km}$ [10].

It is expressed as presented in Equation (28):

$$
L=L_{F S L}+A_{m u}-H_{M G}-H_{B G}-\sum K_{\text {correction }}
$$

where $L=$ The medium path loss $(\mathrm{dB}) ; L_{F S L}=$ The free space loss $(\mathrm{dB}) ; A_{m u}=$ Medium Attenuation (dB); $H_{G M}=$ Mobile station antenna height gain factor; $H_{B G}$ $=$ Base station antenna height gain factor; $K_{\text {correction }}=$ Correction factor gain (such as type of environment, water, surfaces, isolated obstacle etc.).

7) Hata model for urban areas: It is also referred as Okumura-Hata model and widely used radio frequency propagation model for predicting the behaviour of cellular transmissions in built up areas. It has a frequency range of $150-1500$ $\mathrm{MHz}$ with mobile antenna height of 1 - $10 \mathrm{~m}$, base station antenna height 30 $200 \mathrm{~m}$ and link distance $1-10 \mathrm{~km}$. It incorporates both suburban and open areas [10]. It is formulated as presented in Equation (29):

$$
L_{u}=69.55+26.16 \log _{10} f-1.82 \log _{10} h_{B}-C_{h}+\left[44.9-6.55 \log _{10} h_{B}\right] \log _{10} d
$$

for small or medium sized city

$$
C_{H}=0.8+\left(1.1 \log _{10} f-0.7\right) h_{m}-1.56 \log _{10} f
$$

and large cities

$$
C_{H}= \begin{cases}8.29\left[\log _{10}\left(1.54 h_{m}\right)\right]^{2}-1.1, & \text { if } 150 \leq f \leq 200 \\ 3.2\left[\log _{10}\left(11.75 h_{m}\right)\right]^{2}-4.97, & \text { if } 200<f \leq 1500\end{cases}
$$

$L_{u}=$ Path loss in urban areas $(\mathrm{dB}) ; h_{B}=$ Height of base station antenna $(\mathrm{m}) ; h_{m}$ $=$ Height of mobile station antenna $(\mathrm{m}) ; f=$ Frequency of transmission $(\mathrm{MHz})$; $C_{H}=$ Antenna height correction factor; $d=$ Distance between the base and mobile stations $(\mathrm{Km})$.

8) Hata model for open areas is presented in Equation (32) [11]:

$$
L_{o}=L_{u}-4.78\left(\log _{10} f\right)^{2}+18.53 \log _{10} f-40.94
$$

where $L_{o}=$ Path loss in open area $(\mathrm{dB}) ; L_{u}=$ Path loss in urban areas for small sized city $(\mathrm{dB}) ; f=$ Frequency of transmission $(\mathrm{MHz})$.

9) COST231 Extension to Hata Model: A model that is widely used for predicting path loss in mobile wireless system is the COST-231 Hata model [11]. The COST-231 Hata model is designed to be used in the frequency band from $500 \mathrm{MHz}$ to $2000 \mathrm{MHz}$. It also contains corrections for urban, suburban and rural (flat) environments. Although its frequency range is outside that of the measurements, its simplicity and the availability of correction factors has seen it widely used for path loss prediction at this frequency band [12]. The basic equation for path loss in $\mathrm{dB}$ is: 


$$
L=46.3+33.9 \log f-13.82 \log h_{B}-a\left(h_{R}\right)+\left[44.9-6.55 \log h_{B}\right] \log d+c
$$

For suburban or rural environments:

$$
\begin{gathered}
\left(h_{R}\right)=(1.1 \log f-0.7) h_{R}-(1.56 \log f-0.8) \\
c= \begin{cases}0 \mathrm{~dB}, & \text { for mediumcities and suburban areas } \\
3 \mathrm{~dB}, & \text { for metropolitan areas }\end{cases}
\end{gathered}
$$

where, $L=$ Median path loss. Unit: decibel (dB); $f=$ Frequency of Transmission. Unit: megahertz $(\mathrm{MHz}) ; h_{B}=$ Base station antenna effective height. Unit: meter $(\mathrm{m}) ; d=$ Link distance. Unit: Kilometer $(\mathrm{km}) ; h_{R}=$ Mobile station antenna effective height. Unit: meter $(\mathrm{m}) ; a\left(h_{R}\right)=$ Mobile station antenna height correction factor as described in the Hata model for urban areas.

[13] present an investigation on the characteristics of radio propagation, by measurement, at the small town of rural area in Purwokerto Central Java Indonesia. Their results were used to evaluate the accuracy of Okumura Hata and Lee prediction models and to determine the necessary adjustments to these models in order to improve their accuracies. He radiated a $20 \mathrm{dBm}$ signal at $1467 \mathrm{MHz}$ by an omni directional antenna with $5.2 \mathrm{~dB}$ gain. His propagation measurements showed that the received signal strength decreases with distance at the rate of $2.34 \mathrm{~dB}$, while its mean values fall between 10 and $15 \mathrm{~dB}$ below free space prediction with standard deviation of $6.5 \mathrm{~dB}$. Comparing these propagation measurements with Okumura-Hata and Lee prediction models for open area classifications, they discovered that they were in agreement for area coverage of 3 to 10 $\mathrm{Km}$. Also, Okumura-Hata and Lee models gave less path loss prediction for smaller coverage area but propose higher values for larger distances.

Similarly, in Ondo State, the relationship between the line of sight and signal strength of an Ultra High Frequency (UHF) television signals was investigated. The Propagation curves for the signal along different routes were plotted and regression analysis was used to determine the exponential models which can be used to calculate signal strength for a given line of sight at the designated routes in the state [14].

According to Faruk, et al. (2009) [8], Path loss exponent is one of the important parameters in all distance path loss models (log-normal); once it is known for an environment, coverage planning and propagation analysis could be done easily. In their work, log-normal propagation path loss model was used to characterize the path loss parameters in the VHF and UHF frequencies for Ilorin City of Kwara State, Nigeria. Results indicated that the path loss exponent varies from 1.4 to 4.94 with an average value of 2.80 . The work further investigated the behaviour of the TV signals in the same environment in terms of standard deviation and building penetration loss. It is concluded that the standard deviation for Ilorin city is $7.35 \mathrm{~dB}$, the average penetration loss is $11.49 \mathrm{~dB}$ and the path loss intercept at $1 \mathrm{~km}$ at $203.25 \mathrm{MHz}$ and $583.25 \mathrm{MHz}$ is $107.56 \mathrm{~dB}$.

A research in South-Eastern Nigeria by [15], showed that Hata and other empirical models didn't predict the path loss. Instead using the log-normal, the 
outdoor path loss model for Aba urban was obtained as

$$
\operatorname{Lp}(d)=75+31 \log (D)
$$

where $D$ is the ratio of the test distance $\left(d_{i}\right)$ to the reference distance $\left(d_{0}\right)$.

And $75 \mathrm{~dB}$ is the path loss at the reference distance using the free-space model.

GSM signals operating on the frequencies of $900 \mathrm{MHz}$ and $1800 \mathrm{MHz}$, which are the two frequencies used by mobile operators in Nigeria, were investigated in Enugu and Portharcourt. The mean square errors $\left(\mu_{e}\right)$ ranged from $0.8 \mathrm{~dB}$ to $5.04 \mathrm{~dB}$ for Okumura Hata at $900 \mathrm{MHz}$. For COST 231 Hata lied between $1 \leq \mu$ $\leq 15 \mathrm{~dB}$ which is universally accepted [11].

Measurement results of signal strength in UHF band obtained in Idanre Town of Ondo State Nigeria are presented and compared with the results predicted by using the propagation models. A modified COST231-Hata radiowave propagation model was developed and implemented with Matlab GUI (Graphical User Interface) for simulation. The model developed has $93.8 \%$ accuracy [16].

\subsection{Effect of Obstacle on Propagation of Radio Waves}

The free space earlier mention for wave propagation does not really exist, though it serves as a guide for simple computation of signal strength and path loss. Ideally there are obstacles in signal path which greatly influence the predictability of signal strength along any route. These obstacles include buildings, vegetations (foliage) hills.

The density of foliage and the heights of trees that are not uniformly distributed in a forested environment cause variation in the signal reception at different points. The research carried out in Ondo state on the effect of hills on UHF signal propagation showed a marked difference when measurements were taken at two different environments, one with without hills and the other with hills. The field strength decreases rapidly in region with hills [16].

Also, a study by [3], on the effects of rain on the coverage areas of Ondo State Radio/television Corporation (OSRC) UHF Television signals transmitted on Channel $23(487.25 \mathrm{MHz})$ and Channel $25(503.25 \mathrm{MHz})$ in Ondo State revealed that the early part of the dry season recorded the highest signal strength of $75.0 \%$ coverage followed by the onset of raining season at a value of $72.5 \%$. The peak of the raining season recorded the lowest electric field signal strength and coverage of $67.45 \%$.

Furthermore, to understand the degree of interaction, signal strength measurements of the 93.1 MHz frequency modulated Radio located at Federal University of Technology; Akure, Nigeria. The long rice irregular terrain model was used and the losses along the paths were determined. This was compared with the path loss predicted by the irregular terrain model and this was highly correlated. The result offered useful data for developing the contour map of the propagation loss which was developed for the station. It was concluded that with the irregular terrain model predictions can be used for accurate spectrum management in Nigeria [4]. 


\section{Materials and Methods}

\subsection{Materials}

\subsubsection{Study Area}

This research was carried out in Akpabuyo Local Government Area, CRS, Nigeria; one of the few costal states in the Niger Delta region of Nigeria. The study area occupies 20,156 (7782 square miles) square kilometers with coordinates: $5^{\circ} 45^{\prime} \mathrm{N}, 8^{\circ} 30^{\prime} \mathrm{E} / 5.750^{\circ} \mathrm{N}, 8.500^{\circ} \mathrm{E}$. The state has a population of $3,737,517$, with a population density of $190 / \mathrm{Km}^{2}$ ( $480 /$ square miles). The area has distinct environmental characteristics that made Calabar Municipal Council, a challenging location to propagate electromagnetic waves; and therefore recipe for this study. Cross River State has a tropical-humid weather with wet and dry season. It also has an average temperature ranging between $15^{\circ} \mathrm{C}-30^{\circ} \mathrm{C}$; with an annual rainfall between $1300-3000 \mathrm{~mm}$. This condition differs at the high altitude, Obudu plateau. The temperature at this altitude is between $4^{\circ} \mathrm{C}-10^{\circ} \mathrm{C}$. Cross River State has the largest tropically dense forest in Nigeria and in West Africa; which is about 0.85 million hectares containing 1000 square kilometres of mangrove and swamp forest. Its estuary is the largest in Nigeria with a low-energy, shallow coastal waters and shorelines.

These, distinct environmental characteristics made CRS a challenging location to propagate electromagnetic waves; and therefore recipe for this study.

\subsubsection{Digital Cable Television Analyzer (CATV)}

It is radio frequency analyzer, with 24 channels spectrum, ranging between 46 $870 \mathrm{MHz}$ (model: RO.VE.R.-“DLM3-T"). It has a video display screen and corresponding output sound to capture signals from terrestrial radio stations and television stations. CATV measures signal of both radio and TV in $\mathrm{dB}, \mathrm{dB} \mu V$ and $\mathrm{dBmV}$. The equipment works perfectly with $12 \mathrm{~V}$ supply, either from an external battery, or from other available grid supply; and it's capable of powering the pre-amplifier of an antenna system for maximum reception. Figure 2 shows the field strength meter in its operational form displaying a monitored TV channel, while Figures 3-5 are sites where the measurements took place.

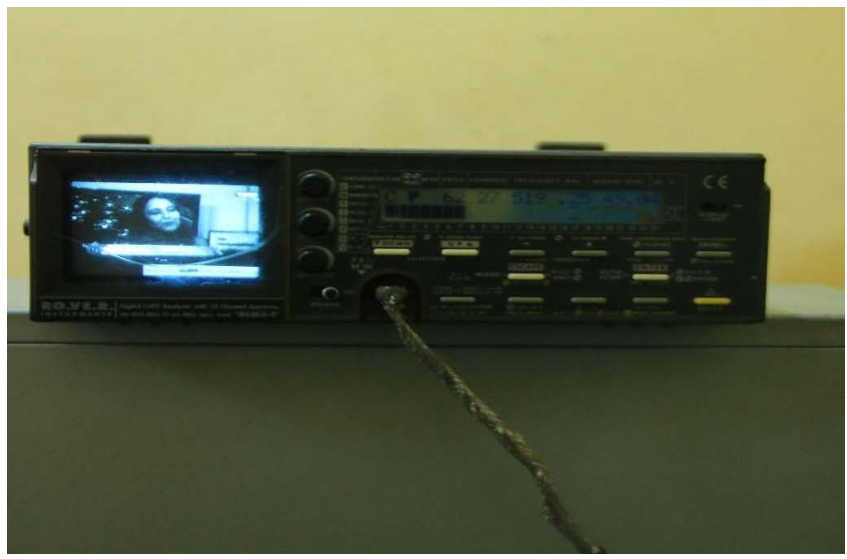

Figure 2. Field strength meter (CATV). 


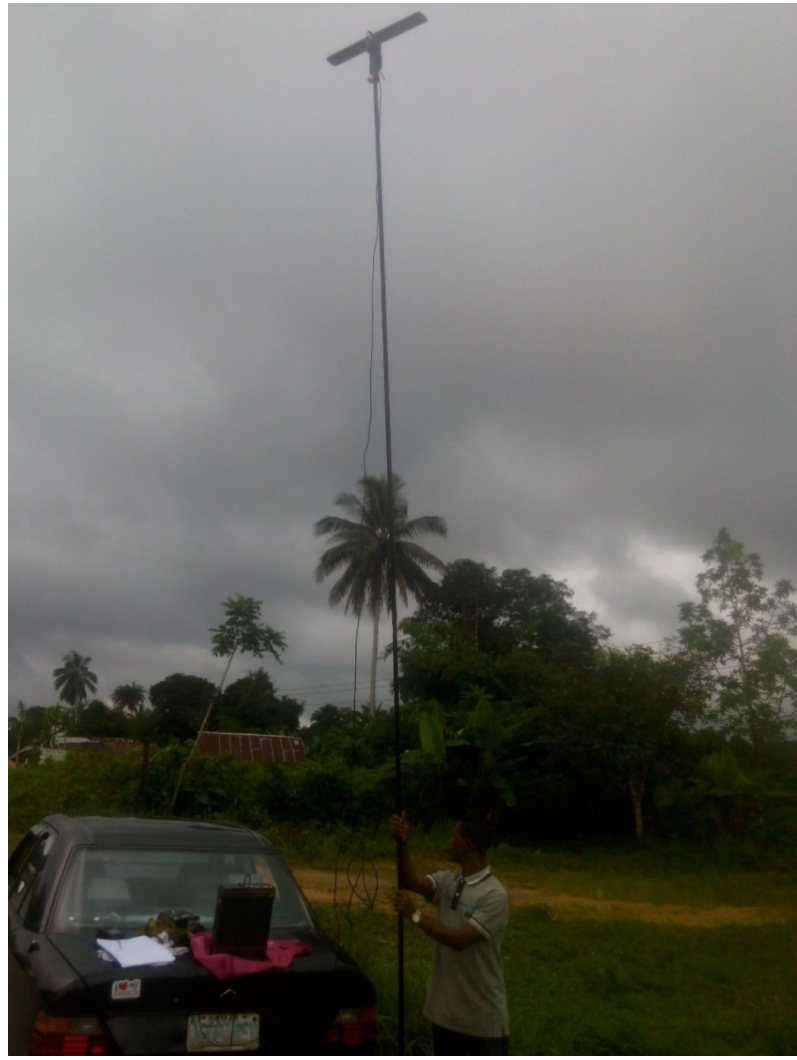

Figure 3. One of the measurement locations (Bakassi L.G.A).

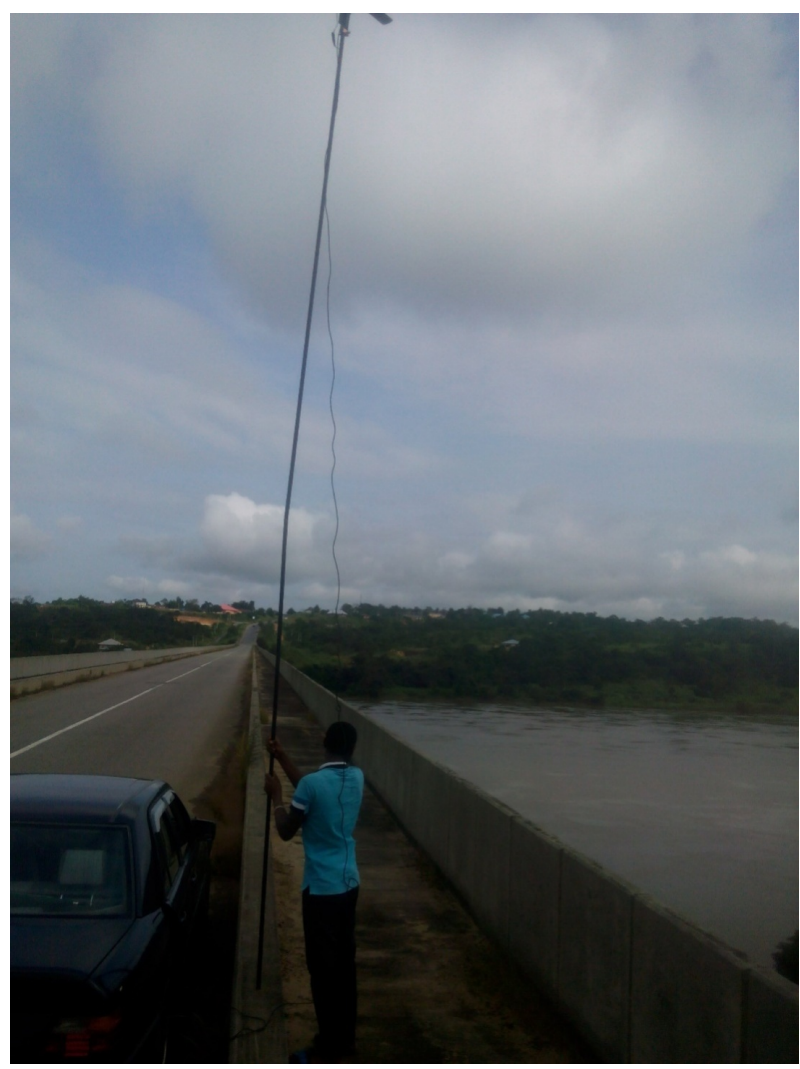

Figure 4. One of the measurements locations (Itighidi bridge). 


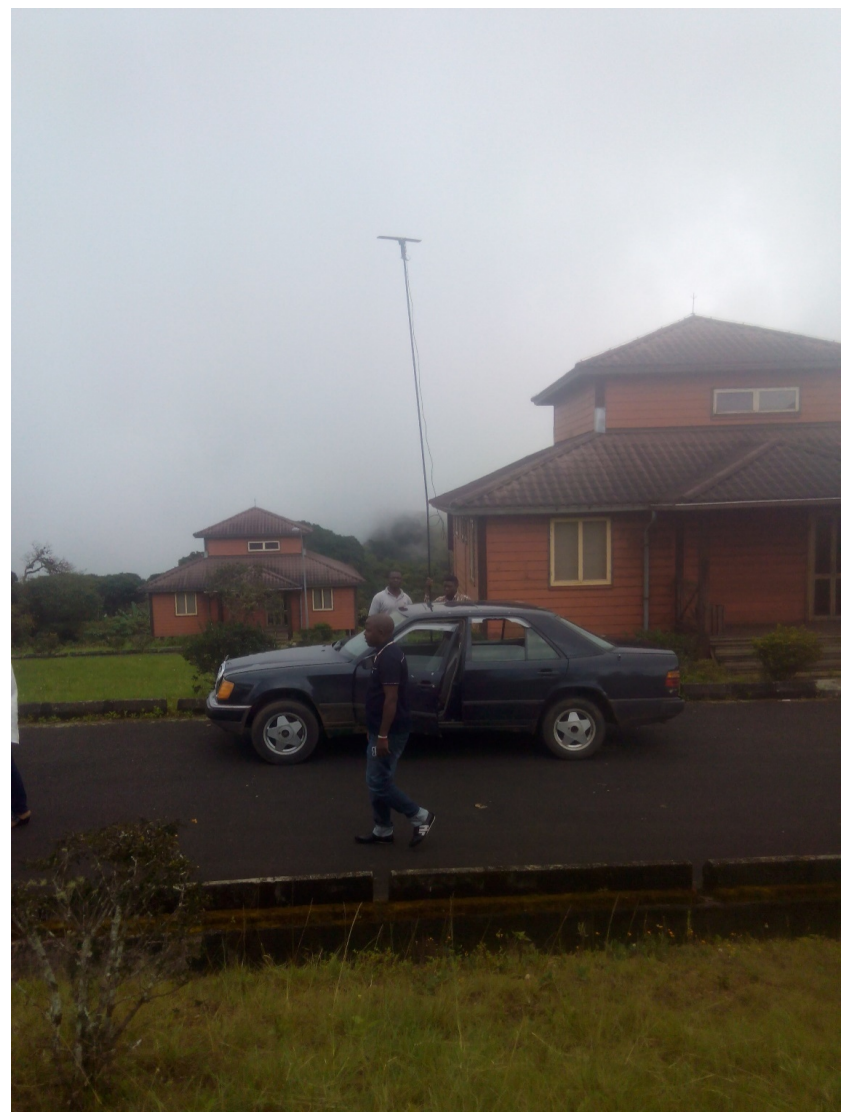

Figure 5. Measurements taken at top hill of Obudu Ranch Resort.

\subsubsection{Receiving Antenna}

An outside television antenna of dimension $59(\mathrm{~L}) \times 8.5(\mathrm{~W}) \times 11(\mathrm{H}) \mathrm{cm}$ on a pole of about $7 \mathrm{~m}$, was used to receive the signal at different locations across the state. Its frequency range spans from $40-860 \mathrm{MHz}$, from channel 1 to channel 69. The antenna has both VHF and UHF gain of $20 \pm 3 \mathrm{~dB}$, with an impedance of $75 \Omega$ and a noise coefficient less than $3 \mathrm{~dB}$. The operational power of this antenna is $3 \mathrm{~W}$ with an output level of $145 \mathrm{~dB}$.

\subsubsection{Global Positioning System}

GPS 72H from Garmin was used to measure the distance from the transmitting antennas to different measuring locations in the state as well as their elevations above sea level.

\subsection{Measurement Procedure}

Measurements were carried out in all the Local Government Areas in the state along motor-able and accessible route. Field or signal strength at various locations were examined both at the UHF and VHF bands, with special attention given to some reliefs along the measuring route. This was made possible by large contour map of the state. At each location, the signal strength of various stations alongside the elevation above sea level and coordinates in northings and eastings were measured. Measurements of the signal strength were measured first at 
premises of the stations; also referred to as the near field signal, before subsequent measurements at other locations.

\section{Results and Discussion}

\section{Analysis of Results from Akpabuyo Local Government Area}

Figure 6 is the Map of Akpabuyo Local Government Area, showing points of measurements. Figure 7, is the plot of signal strength levels from Channel 11 against distance in Akpabuyo Local Government Area. Figure 8 is the plot of path loss in Channel 11 against distance in Akpabuyo Local Government Area. Figure 9 is the plot of calculated path loss and path loss models in Channel 11 against distance in Akpabuyo Local Government Area. Figure 10, is plot of signal strength from Channel 27 against distance in Akpabuyo Local Government Area. Figure 11 is plot of path loss in Channel 27 against distance in Akpabuyo Local Government Area. Figure 12 is plot of calculated path loss and other models in Channel 27 against distance in Akpabuyo Local Government Area. Figure 13 is plot of signal strength from Channel 45 against distance in Akpabuyo Local Government Area. Figure 14 is plot of path loss in channel 45 gainst distance in Akpabuyo Local Government Area, and Figure 15 is plot of calculated path loss and other models in Channel 45 against distance in Akpabuyo Local Government Area. Similarly, Table 1 tabulates the descriptive statistics of path loss models in Channel 27 in Akpabuyo Local Government Area while Table 2 presents the descriptive statistics of path loss models in Channel 45 in Akpabuyo Local Government Area.

Table 1. Descriptive statistics of path loss models in channel 11 at Akpabuyo.

\begin{tabular}{cccccc}
\hline Statistics & Field Data & Cost231Hata & Egli & Okuruma & Free Space \\
\hline Min & 47.7 & 87.52 & 58.27 & 147.2 & 285.7 \\
Max & 82.6 & 99.73 & 66.27 & 152.9 & 289.7 \\
Mean & 66.08 & 93.96 & 62.49 & 150.2 & 287.8 \\
Median & 66.2 & 94.51 & 62.85 & 150.5 & 288 \\
Mode & 47.7 & 87.52 & 58.27 & 147.2 & 285.7 \\
Std & 8.173 & 3.365 & 2.205 & 1.584 & 1.102 \\
\hline
\end{tabular}

Table 2. Descriptive statistics of path loss models in channel 27 at Akpabuyo.

\begin{tabular}{cccccc}
\hline Statistics & Field Data & Cost231Hata & Egli & Okuruma & Free Space \\
\hline Min & 40.7 & 110.2 & 33.95 & 159.2 & 303.6 \\
Max & 43.1 & 116.2 & 37.86 & 164.9 & 305.5 \\
Mean & 41.94 & 113.2 & 35.92 & 162.2 & 304.6 \\
Median & 42 & 113.2 & 35.9 & 162.4 & 304.5 \\
Mode & 42.1 & 110.2 & 33.95 & 159.2 & 303.6 \\
Std & 0.5384 & 1.617 & 1.058 & 1.579 & 0.5288 \\
\hline
\end{tabular}




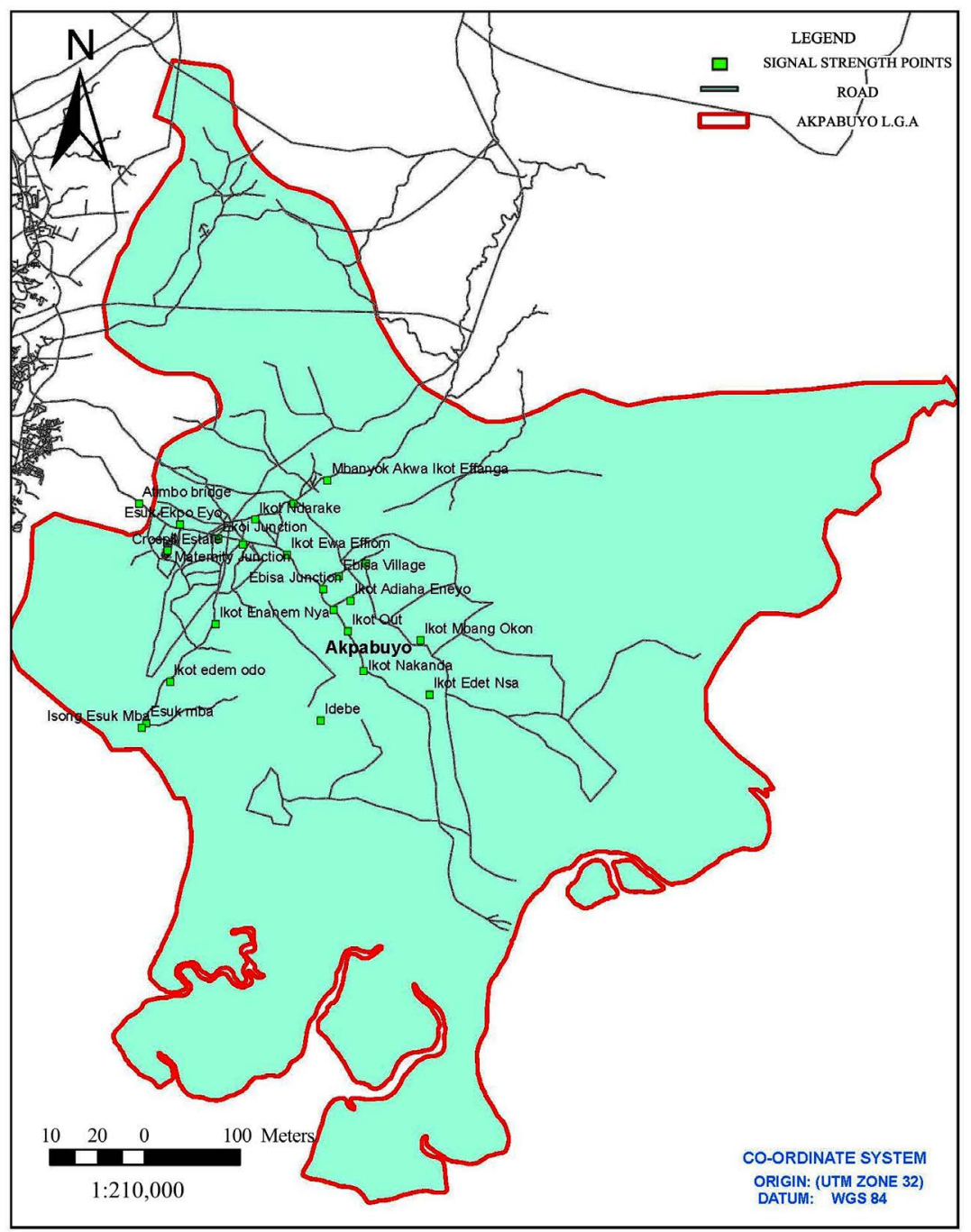

Figure 6. Map of Akpabuyo showing points of measurement.

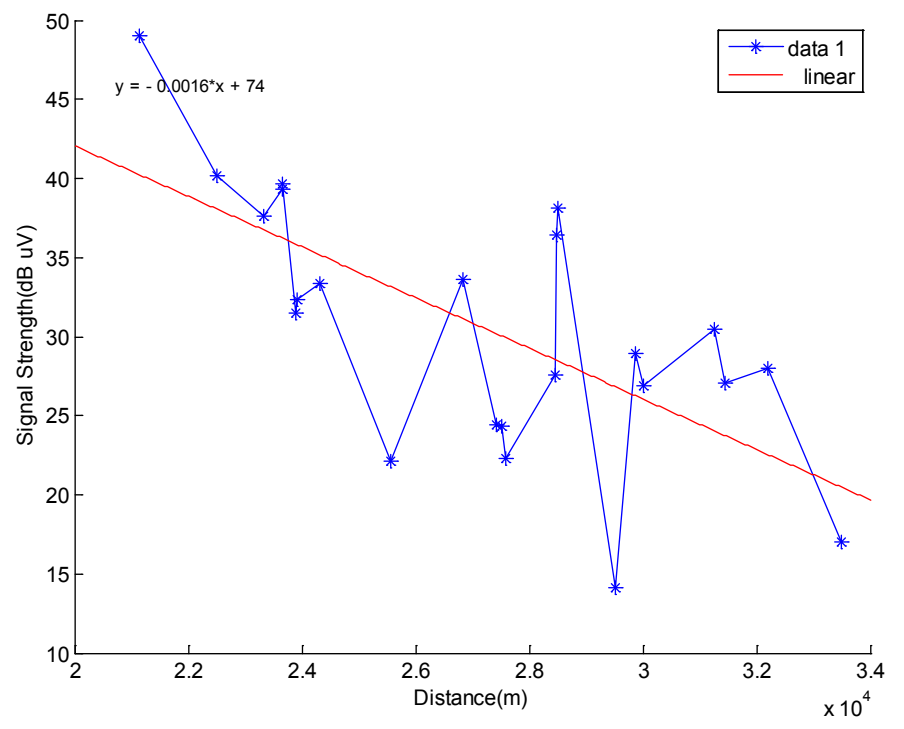

Figure 7. Plot of signal strength from Channel 11 against distance in Akpabuyo. 


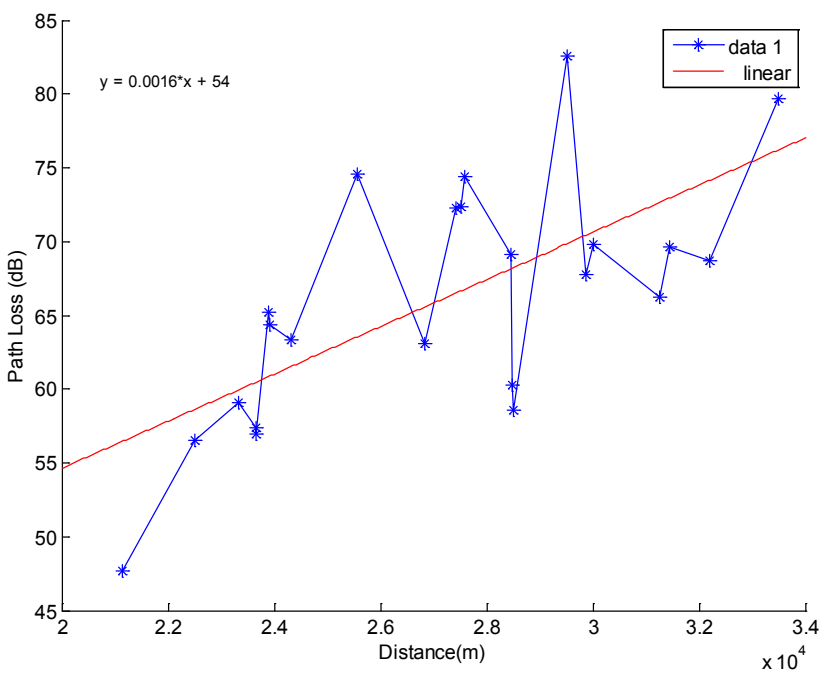

Figure 8. Plot of calculated path loss and other models in Channel 11 at Akpabuyo.

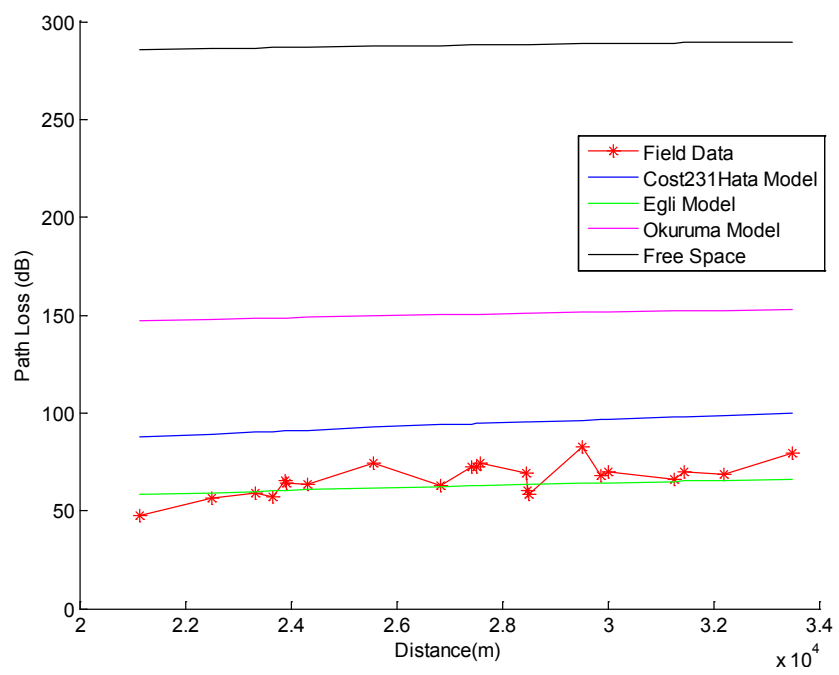

Figure 9. Plot of calculated path loss and models in Channel 11 at Akpabuyo.

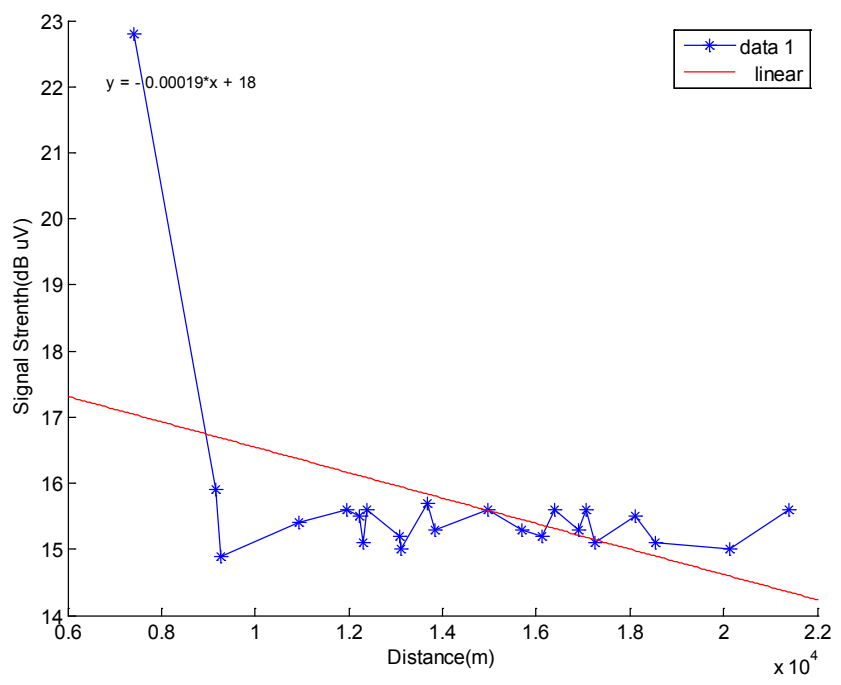

Figure 10. Plot of signal strength from Channel 27 against distance. 


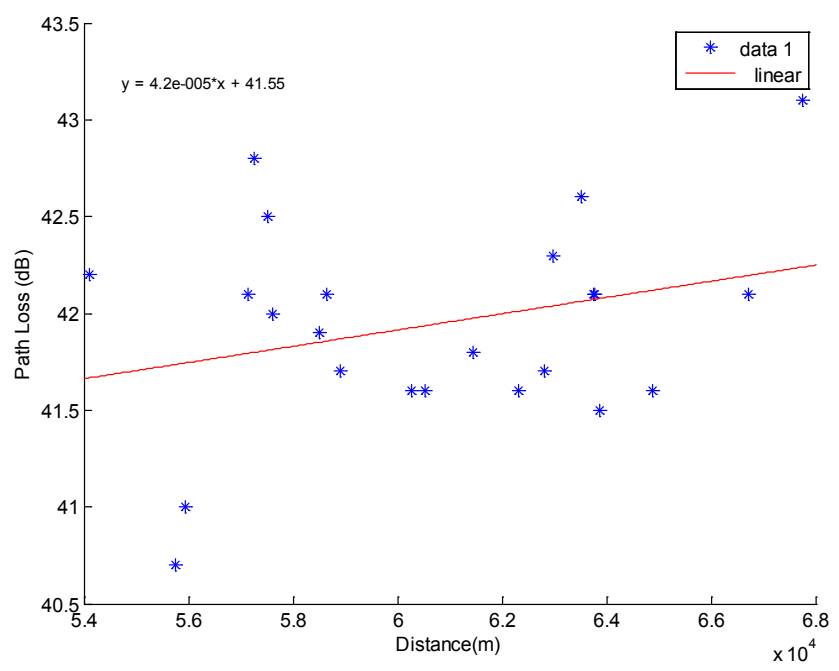

Figure 11. Plot of calculated path loss in Channel 27 against distance at Akpabuyo.

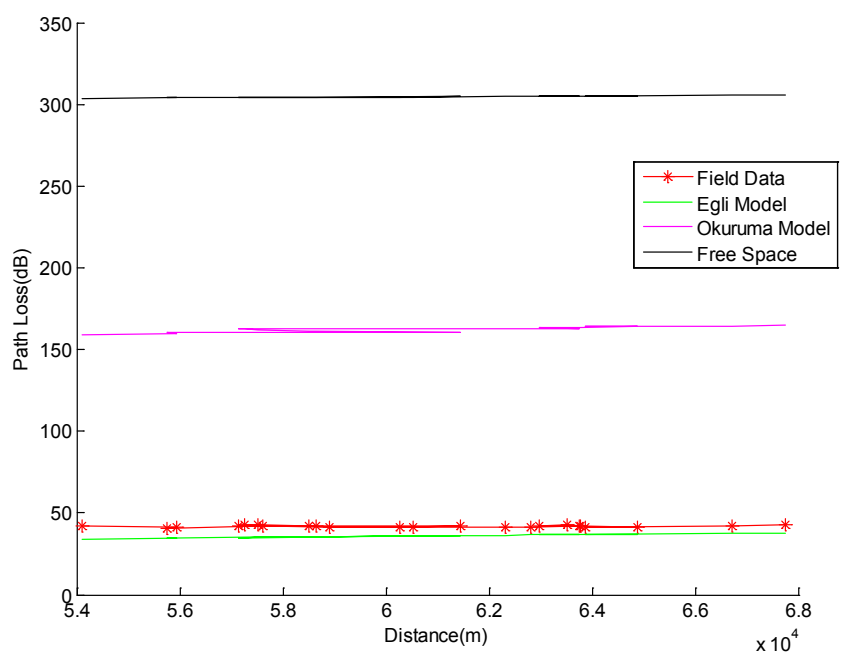

Figure 12. Plot of calculated path loss in Channel 27 against distance at Akpabuyo.

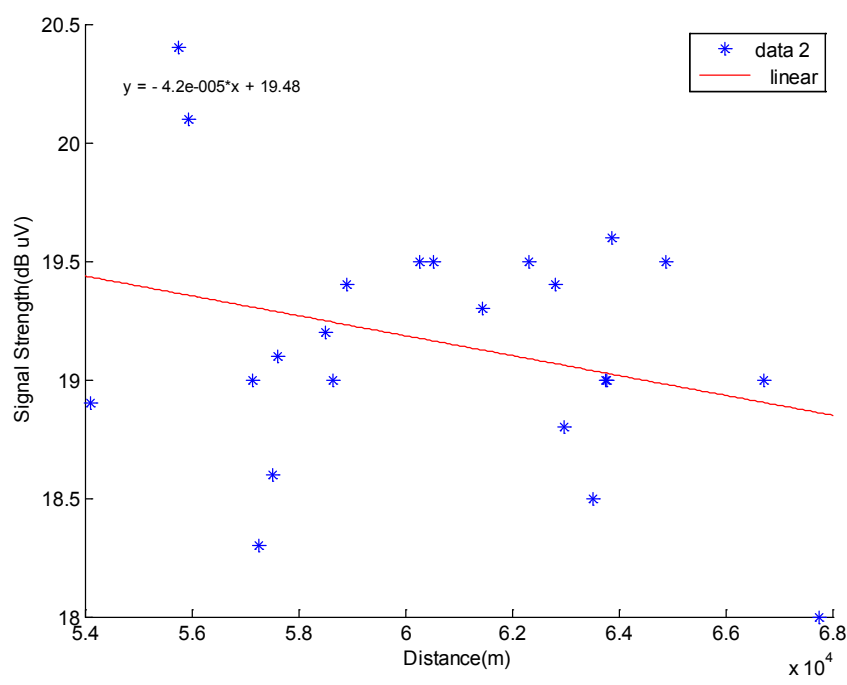

Figure 13. Plot of signal strength from Channel 45 against distance at Akpabuyo. 


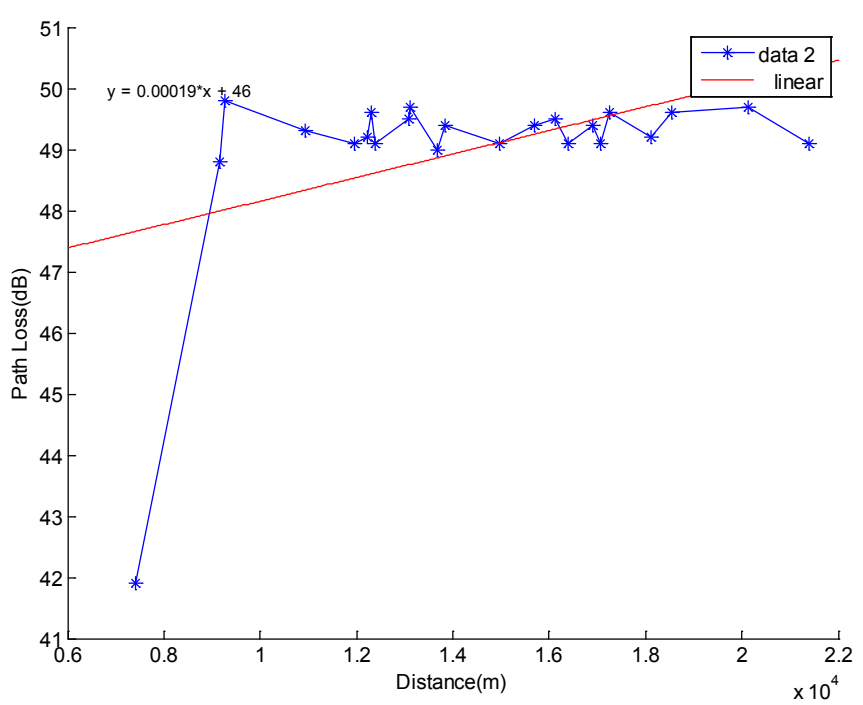

Figure 14. Plot of calculated path loss in Channel 45 against distance at Akpabuyo.

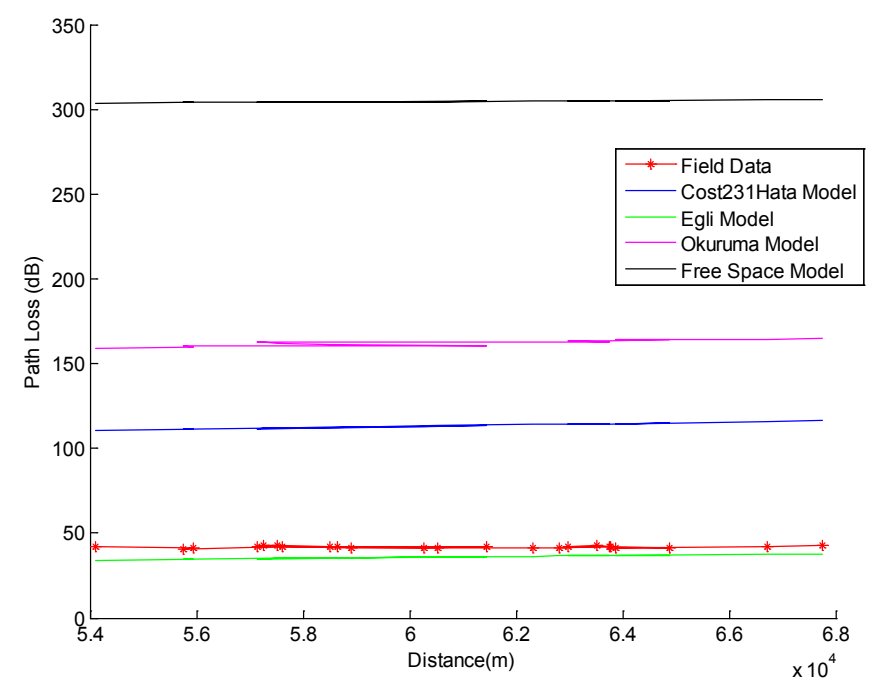

Figure 15. Plot of calculated path loss and other models against distance in Channel 45 at Akpabuyo.

Measurements taken from Akpabuyo L.G.A. showed the following results: the signal strength received from VHF Channel 11 ranged from $20 \mathrm{~dB} \mu \mathrm{V}$ to $49 \mathrm{~dB} \mu \mathrm{V}$. From recorded empirical statistics from the study, $50 \%$ of the area received signal from this station above $30 \mathrm{~dB} \mu \mathrm{V}$, while other regions have signals below this value; representing the fringe zone of the frequency. The Egli model strongly agreed with the calculated path loss from the field data as shown in the figure. Poor signal in this area could be attributed to obstructions along the propagation path since the signal must pass through Calabar city located along the line of sight of the signal before getting to residents in Akpabuyo Local Government Area.

There was no video traffic at all from Channels 27 and 45 in this area; although the study recorded a steady fluctuation between $17 \mathrm{~dB} \mu \mathrm{V}$ and $19 \mathrm{~dB} \mu \mathrm{V}$ 
from both propagating stations. This means that the signal completely faded off before getting to residents of this area; as the transmitter was monitored and confirmed not to be off.

\section{Summary}

\subsection{Summary}

The Cross River State Broadcasting Corporation (CRBC) television, transmitting at Channel 11, of frequency $219.25 \mathrm{MHz}$, has its near field signal strength coverage in some parts of Akpabuyo Local Government Area. Minority of the residents in this part of the rural location fell within the secondary coverage zone, while others were in the fringe zone. The calculated path loss, when compared with standard path loss models in areas where there was signal, closely agreed with predictions indicated in Egli's model; in terms of path loss prediction. Channel 27 signal, at $519 \mathrm{MHz}$, has very scanty signal in many parts of Akpabuyo Local Government Area. It is interesting to note that residents in this area depend only on other Nigerian national electronic media to receive information; including news relating to the state they reside in.

Channel 27 signal at $519 \mathrm{MHz}$, has very weak signal coverage in Akpabuyo Local Government Area; with signal strength below to $50 \mathrm{~dB}$. Also, Egli's model closely agreed with the calculated path loss in the regions where this signal was received. Furthermore, AKBC channel 45 signal from Uyo was scanty received. Received signal strength dropped to as low as $13 \mathrm{~dB}$ in many parts of the rural area. Egli's model was the only path-loss model that closely predicted the path loss at this frequency in this rural setting. Details are as presented in Figures 8-15 and Table 1 and Table 2.

\subsection{Conclusion}

Terrestrial broadcasting, as monitored by this study, on the coverage of one of the Local Government Area (Akpabuyo Local Government Area), using one spot transmission out-let is unachievable. This is predicated on the fact that the state's topography is naturally undulating; thus, affecting the smooth travelling of electromagnetic signals. The state's broadcasting stations, both at UHF and VHF channels do not transmit successfully across this densely forest (rural) setting. The state, therefore, is poorly covered in terms of television signal. Remedial measures such as installing Repeater stations at different locations as signal booster are strongly recommended.

\section{Conflicts of Interest}

The authors declare no conflicts of interest regarding the publication of this paper.

\section{References}

[1] United Nations (2011) Global Development and Goal Indicator, 2011 Global Digital 
Divide.

[2] Kenedy and Davis (1999) Electronic Communication Systems. Fourth Edition, 236.

[3] Ajewole, M.O., Akinbolati, A., Adediji, A.T. and Ojo, J.S. (2014) Precipitation Effect on the Coverage Areas of Terrestrial UHF Television Stations in Ondo State, Nigeria. International Journal of Engineering and Technology, 4, 524-535.

[4] Oyetunji, S.A. (2013) Determination of Propagation Path Loss and Contour Map for FUTA FM Radio Federal University of Technology, Akure Nigeria. IOSR Journal of Electronics and Communication Engineering, 6, 4-9.

https://doi.org/10.9790/2834-0630409

[5] Ostrovsky, L.A. and Potapov, A.I. (2002) Modulated Waves: Theory and Application. Johns Hopkins University Press, Baltimore.

[6] Griffiths, D.J. (1999) Introduction to Electrodynamics. 3rd Edition, Prentice Hall, Upper Saddle River, 301-303.

[7] Kenedy and Davies (1999) Electronic Communication Systems. 4th Edition.

[8] Faruk, N., Ayeni, A. and Adediran, A. (2013) Characterization of Propagation Path Loss at Vhf/Uhf Bands for Ilorin City, Nigeria. Nigerian Journal of Technology, 32, 253-265.

[9] Egli, J.J. (1957) Radio Propagation above $40 \mathrm{MC}$ over Irregular Terrain. Proceedings of the IRE, 45, 1383-1391. https://doi.org/10.1109/JRPROC.1957.278224

[10] Seybold, J.S. (2005) Introduction to RF Propagation. John Wiley and Sons, Hoboken, 144-146. https://doi.org/10.1002/0471743690

[11] Ogbulezie, J., Onuu, M., Ushie, J. and Usibe, B. (2013) Propagation Models for GSM 900 and $1800 \mathrm{MHz}$ for Port Harcourt and Enugu, Nigeria. Network and Communication Technologies, 2. https://doi.org/10.5539/nct.v2n2p1

[12] Mardeni, R. and Kwan, K.F. (2010) Optimization of Hata Propagation Prediction Model in Suburban in Malaysia. Progress in Electromagnetics Research C, 13, 91-106. https://doi.org/10.2528/PIERC10011804

[13] Nadir, Z., Elfadhil, N. and Touati, F. (2009) Path Loss Determination, Using Okumura-Hata Model and Spline Interpolation for Missing Data for Oman. Proceedings of the World Congress on Engineering, London, 2-4 July 2008.

[14] Oke, M. and Raji, R. (2014) Exponential Models of Signal Strength of a Television Station in Nigeria. International Journal of Mathematics and Statistics Studies, 2, 45-54.

[15] Nwalozie, G.C., Ufoaroh, S.U., Ezeagwu, C.O. and Ejiofor, A.C. (2014) Path Loss Prediction for GSM Mobile Networks for Urban Region of Aba, South-East Nigeria. International Journal of Computer Science and Mobile Computing, 3, 267-281.

[16] Oluwole, F.J. and Olajide, O.Y. (2013) Radio Frequency Propagation Mechanisms and Empirical Models for Hilly Areas. International Journal of Electrical and Computer Engineering, 3, 372-376. https://doi.org/10.11591/ijece.v3i3.2519 